DOI

ARTICULO ORIGINAL

\title{
Estudio descriptivo de pacientes con EPOC asistidos en medicina interna del Hospital Pasteur de Montevideo: características demográficas y comorbilidades
}

Selene Correa. ORCID: 0000-0002-2388-9063 Internista. Nefróloga. Profesor Adjunta Clínica Médica. Hospital Pasteur.

UdelaR.

Mariana Gonzaléz. ORCID: 0000-0001-5799-1245 Internista. Ex Asistente Clínica Médica. Hospital Pasteur. UdelaR.

Sofía de Betolaza.

ORCID: 0000-0001-6843-2633 Internista. Asistente Clínica Médica.

Hospital Pasteur. UdelaR.

Cecilia Spiess

ORCID: 0000-0002-0175-2760 Residente Medicina Interna. Clínica Médica 2. Hospital Pasteur. UdelaR.

Patricia Perera. ORCID: 0000-0003-2536-7709 Residente Medicina Interna. Clínica Médica 2. Hospital Pasteur. UdelaR.

Santiago Algorta

ORCID: 0000-0002-7613-3809 Residente Medicina Interna. Clínica Médica 2. Hospital Pasteur. UdelaR.

Mabel Goñi

ORCID: 0000-0001-8593-6569 Internista. Intensivista. Profeso Director Clínica Médica. Hospital Pasteur. UdelaR.

\section{Descriptive study of patients with COPD assisted in internal medicine of the Hospital Pasteur of Montevideo: demographic characteristics and comorbidities}

\section{Estudo descritivo de pacientes com DPOC atendidos em medicina interna do Hospital Pasteur de Montevidéu: características demográficas} e comorbidades

Resumen. Introducción: La EPOC es una enfermedad de alta prevalencia, base inflamatoria y compromiso sistémico, asociada a múltiples comorbilidades que afectan sinérgicamente la calidad de vida y pronóstico de los pacientes. Objetivo: Describir las principales características demográficas de una población de pacientes con EPOC. Valorar las comorbilidades más frecuentes y su relación con la enfermedad. Materiales y métodos: Estudio observacional, descriptivo y transversal desde el $1^{\circ}$ de octubre de 2015 al 31 de diciembre de 2016 . Criterios de inclusión: ambos sexos, mayores de 40 años, clínica y factores de riesgo compatibles o diagnóstico previo de EPOC. Criterios de exclusión: deterioro cognitivo moderado-severo, pacientes postrados, embarazadas, tuberculosis pulmonar activa, enfermedades neuromusculares. Muestreo realizado por conveniencia. Resultados: 61 pacientes captados, se confirmó EPOC por espirometría en 42 . Edad media 63 años $( \pm 11)$ y predominó en el sexo masculino $(24,57.1$ \%). La mayoría tenía bajo nivel de instrucción (primaria incompleta en 15 pacientes, 35.7\%). El tabaquismo se constató en el 100\% como factor de riesgo, alcoholismo en 14 pacientes (33.3\%) y obesidad en 10 (23.8\%), con un IMC máximo de 41 y una media de $25.79 \pm 6.83 \mathrm{Kg} / \mathrm{m} 2$. De las comorbilidades indagadas, la HTA es la más frecuente, le siguen las enfermedades respiratorias asociadas (asma, intersticiopatías y SAHOS) en 6 pacientes, 14,3\%. Otras comorbilidades como dislipemia (8 pacientes, 19\%), diabetes mellitus (6 pacientes, $14.3 \%)$, cardiopatía isquémica (4 pacientes, 9.5\%), CBP (3 pacientes, $7.1 \%)$, infección por VIH (2 pacientes, $4.8 \%$ ) y enfermedad renal crónica (1 paciente, $2.4 \%$ ) fueron también analizadas. Conclusiones: La edad media y el sexo coinciden con los datos internacionales. Predominó el nivel de instrucción bajo y medio, lo que tiene implicancias en la comprensión de la enfermedad y las comorbilidades. El principal factor de riesgo para el desarrollo de la enfermedad es el tabaquismo. El 100\% de los pacientes tenían comorbilidades. La HTA y el alcoholismo son las más frecuentes. Existe una relación significativa entre EPOC y la presencia de alteraciones endocrino-metabólicas como obesidad, dislipemia y diabetes. La presencia de otras enfermedades respiratorias constituyen un desafío diagnóstico. La relación con CBP es siempre estrecha por compartir el tabaquismo como agente etiológico. El número y tipo de comorbilidades se relaciona con el pronóstico vital a corto plazo cuando los pacientes son hospitalizados, en una exacerbación de la EPOC.

Palabras clave: EPOC, comorbilidades

Abstract. Introduction: COPD is a disease of high prevalence, inflammatory base and systemic involvement, associated with multiple comorbidities that synergistically affect the quality of life 
and prognosis of patients. Objective: To describe the main demographic characteristics of a population of patients with COPD. Assess the most frequent comorbidities and their relationship with the disease. Materials and methods: Observational, descriptive and cross-sectional study from October 1, 2015 to December 31, 2016. Inclusion criteria: both sexes, over 40 years of age, clinical and compatible risk factors or previous diagnosis of COPD. Exclusion criteria: moderatesevere cognitive impairment, prostrate patients, pregnant women, active pulmonary tuberculosis, neuromuscular diseases. Sampling done for convenience. Results: 61 patients received, COPD was confirmed by spirometry in 42 . Mean age was 63 years $( \pm 11)$ and it predominated in males $(24,57.1 \%)$. The majority had low educational level (incomplete primary in 15 patients, 35.7\%). Smoking was found in $100 \%$ as a risk factor, alcoholism in 14 patients (33.3\%) and obesity in 10 (23.8\%), with a maximum BMI of 41 and a mean of $25.79 \pm 6.83 \mathrm{Kg} / \mathrm{m} 2$. Of the comorbidities investigated, hypertension is the most frequent, followed by associated respiratory diseases (asthma, interstitial diseases and OSAHS) in 6 patients, 14.3\%. Other comorbidities such as dyslipidemia (8 patients, 19\%), diabetes mellitus (6 patients, 14.3\%), ischemic heart disease (4 patients, 9.5\%), PBC (3 patients, 7.1\%), HIV infection (2 patients, 4.8\%) and chronic kidney disease (1 patient, $2.4 \%$ ) were also analyzed. Conclusions: Average age and sex coincide with international data. Low and medium level of education predominated, which has implications in the understanding of the disease and comorbidities. The main risk factor for the development of the disease is smoking. $100 \%$ of patients had comorbidities. HTA and alcoholism are the most frequent. There is a significant relationship between COPD and the presence of endocrinemetabolic disorders such as obesity, dyslipidemia and diabetes. The presence of other respiratory diseases is a diagnostic challenge. The relationship with CBP is always close because of sharing smoking as an etiological agent. The number and type of comorbidities are related to short-term vital prognosis when patients are hospitalized, in an exacerbation of COPD.

Keywords: COPD, comorbidities

Resumo. Introdução: A DPOC é uma doença de alta prevalência, base inflamatória e envolvimento sistêmico, associada a múltiplas comorbidades que afetam sinergicamente a qualidade de vida e o prognóstico dos pacientes. Objetivo: Descrever as principais características demográficas de uma população de pacientes com DPOC. Avaliar as comorbidades mais frequentes e sua relação com a doença. Materiais e métodos: Estudo observacional, descritivo e transversal, de $1^{\circ}$ de outubro de 2015 a 31 de dezembro de 2016. Critérios de inclusão: ambos os sexos, maiores de 40 anos, fatores de risco clínicos e compatíveis ou diagnóstico prévio de DPOC. Critérios de exclusão: comprometimento cognitivo moderado-grave, pacientes prostrados, gestantes, tuberculose pulmonar ativa, doenças neuromusculares. Amostragem feita por conveniência. Resultados: dos 61 pacientes atendidos, a DPOC foi confirmada pela espirometria em 42 . A idade média foi de 63 anos $( \pm 11)$ e predominou no sexo masculino $(24,57,1 \%)$. A maioria tinha baixo nível educacional (primário incompleto em 15 pacientes, 35,7\%). O tabagismo foi encontrado em $100 \%$ como fator de risco, alcoolismo em 14 pacientes (33,3\%) e obesidade em 10 (23,8\%), com IMC máximo de 41 e média de 25,79 $\pm 6,83 \mathrm{Kg} / \mathrm{m} 2$. Das comorbidades investigadas, a hipertensão arterial é a mais frequente, seguida das doenças respiratórias associadas (asma, doenças intersticiais e SAHOS) em 6 pacientes, 14,3\%. Co-morbidades e dislipidemia (8 pacientes, 19\%), diabetes mellitus (6 pacientes, 14,3\%), doença isquémica cardíaca (4 pacientes, 9,5\%), PFC (3 pacientes, 7,1\%), infecção por VIH (2 pacientes, 4,8\%) e doença renal crônica (1 paciente, $2,4 \%$ ) também foram analisados. Conclusões: Idade média e sexo coincidem com dados internacionais. Baixa e média escolaridade predominou, o que tem implicações na compreensão da doença e comorbidades. O principal fator de risco para o desenvolvimento da doença é o tabagismo. $100 \%$ dos pacientes tinham comorbidades. A HTA e o alcoolismo são os mais frequentes. Existe uma relação significativa entre a DPOC e a presença de distúrbios endócrino-metabólicos, como obesidade, dislipidemia e diabetes. A presença de outras doenças respiratórias é um desafio diagnóstico. A relação com o CBP está sempre próxima, por compartilhar o tabagismo como agente etiológico. O número e o tipo de comorbidades estão relacionados ao prognóstico vital de curto prazo quando os pacientes são hospitalizados, em uma exacerbação da DPOC.

Palavras-chave: DPOC, comorbidades 


\section{Introducción}

Los pacientes con Enfermedad Pulmonar Obstructiva Crónica (EPOC) tienen mayor frecuencia de comorbilidades que la población general (1)

La EPOC se caracteriza por una limitación persistente del flujo aéreo, progresiva asociada a una respuesta inflamatoria crónica exagerada de las vías aéreas y pulmonares frente a gases y partículas nocivas. La limitación del flujo aéreo ocurre por afectación de la pequeña vía aérea y la destrucción del parénquima pulmonar ${ }^{(2)}$

Es una enfermedad de alta prevalencia, base inflamatoria y compromiso sistémico, asociada a múltiples comorbilidades que afectan sinérgicamente la clase funcional, la calidad de vida y pronóstico de los pacientes. Las comorbilidades son las enfermedades asociadas a la EPOC y no forman parte de la historia natural de ella ${ }^{(3)}$

El aumento en la esperanza de vida y el tratamiento adecuado de la enfermedades crónicas ha hecho que en los últimos años la población esté afectada en forma simultánea por varias patologías que interactúan entre sí lo que dificulta el diagnóstico y el tratamiento y empeora el pronóstico, por lo que algunos consideran los términos pluripatología o multi -morbilidad más conveniente que el de comorbilidad ${ }^{(1)}$

Se conoce poco de como las condiciones crónicas se presentan con ciertos patrones de multimorbilidad. En un estudio sobre patrones globales de multimorbilidad donde se analizaron los datos procedentes del proyecto europeo Collaborative Research on Ageing y datos de la Organización Mundial de la Salud, se obtuvieron 41909 pacientes adultos mayores de 50 años. La prevalencia de multimorbilidad fue alta. Se identificaron varios patrones en diferentes países: "cardiorespiratorio" (angor, asma y EPOC), "metabólico" (diabetes, obesidad e hipertensión arterial) y el patrón "mentaarticular" (artritis y depresión). El conocimiento de estos patrones permite desarrollar acciones preventivas para disminuir la prevalencia y dar lugar a enfoques integrales para el manejo de algunas condiciones concurrentes ${ }^{(4,5)}$.

Así las enfermedades crónicas incluidas las patologías cardiovasculares, respiratorias y metabólicas ocurren asociadas, están en aumento en los países en desarrollo y tienen alto costo social y económico. El costo de las enfermedades crónicas individuales aumenta exponencialmente en pacientes con dos o más comorbilidades. Casi la mitad de las personas mayores de 65 años tienen al menos tres comorbilidades y una quinta parte tienen cinco o más (1). Las enfermedades cardiovasculares y las neoplasias son las que generan mayor mortalidad, en trabajos realizados en la población ambulatoria se estima que los pacientes con EPOC tienen un riesgo ajustado 2 a 5 veces superior de tener cardiopatía isquémica que los pacientes sin la EPOC independientemente de otros factores de riesgo vascular incluyendo el tabaquismo (6). Además en las semanas posteriores a la exacerbación de la EPOC aumenta el riesgo de presentar un evento isquémico, porque aumentan los parámetros inflamatorios, la rigidez arterial y la agregación plaquetaria ${ }^{(7)}$.

Existe evidencia que el volumen expirado en el primer segundo (VEF1) deteriorado es un marcador de morbimorbilidad cardiovascular. El aumento de la rigidez arterial se relaciona con la gravedad de la obstrucción al flujo aéreo ${ }^{(8,9)}$. La EPOC se asocia a insuficiencia cardíaca en más del $20 \%$ de los pacientes ${ }^{(5)}$ y los pacientes con las dos patologías tienen menor capacidad de esfuerzo y mayor mortalidad (10)

La cardiopatía isquémica es un conjunto de síntomas y signos que ocurren por disminución del aporte de oxígeno al miocardio en relación con los requerimientos metabólicos de éste, ya sea por disminución del flujo coronario por debajo del umbral mínimo necesario o por aumento de los requerimientos o disminución del volumen de oxígeno trasportado ${ }^{(11,12)}$.

La hipertensión arterial (HTA) es un desorden hemodinámico que puede provocar enfermedad cardiovascular, así cuanto menores sean las cifras de la presión arterial menor es el riesgo cardiovascular y la morbimortalidad. Se considera hipertensos los pacientes con valores de presión arterial sistólica mayor a $140 \mathrm{mmHg}$ y presión arterial diastólica mayor a $90 \mathrm{mmHg}{ }^{(13)}$. La HTA tiene una prevalencia en Uruguay de 37\% según la Sociedad Uruguaya de Hipertensión Arterial y de $38,7 \%$ según la Encuesta Nacional de Factores de Riesgo de Enfermedades Crónicas no Trasmisibles realizada en el año $2013^{(14)}$ Hay varios estudios que demuestran que la HTA es la comorbilidad más frecuente ${ }^{(15,16)}$.

La EPOC cursa con inflamación sistémica, caracterizada por niveles plasmáticos elevados de citoquinas pro inflamatorias (Interleuquinas 6 y 9, Factor de necrosis tumoral $₫$ ) proteína C reactiva, estrés oxidativo, y activación de células inflamatorias, en la enfermedad estable y en la exacerbación. También se ha demostrado que cuanto más alto es la obstrucción del flujo aéreo, más altos son los marcadores inflamatorios ${ }^{(17)}$ 
Este grado de inflamación sistémica permanente, asociado al envejecimiento acelerado, la pérdida de tejido conectivo, el aumento de la rigidez arterial y el compartir ciertos factores de riesgo como el tabaquismo, la edad, el sexo y el sedentarismo pueden ser el nexo entre el EPOC y la hipertensión arterial ${ }^{(18)}$

El alcoholismo según la Organización Mundial de la Salud es una sustancia psicoactiva con propiedades causantes de dependencia, el consumo nocivo conlleva una pesada carga social y económica para las sociedades. La prevalencia del consumo de problemático de alcohol en Uruguay en el año 2013 en la población de 25 a 65 años es de 8,1\%, mayor en hombres (12,9\%) que en mujeres $(1,8 \%)^{(14)}$.

Diversos estudios han demostrado la asociación entre el abuso prolongado del alcohol y el padecimiento de enfermedades crónicas como cirrosis, hepatitis, anemia malnutrición y depresión entre otras ${ }^{(14)}$. La asociación entre el consumo de alcohol y la EPOC no es totalmente conocida ${ }^{(19)}$. El alcohol no fue encontrado como un marcador independiente de hospitalización relacionada con la EPOC ${ }^{(20)}$.

El sobrepeso y la obesidad se determina a través del índice de masa corporal (IMC), obtenido por la relación entre el peso y la talla al cuadrado. El sobrepeso se define con el IMC $\geq 25 \mathrm{Kg} / \mathrm{m} 2$ $\mathrm{y}<\mathrm{a} 30 \mathrm{~kg} / \mathrm{m} 2$ y la obesidad con el IMC $\geq 30 \mathrm{Kg} / \mathrm{m} 2{ }^{(14)}$. En Uruguay la prevalencia de sobrepeso y la obesidad en personas entre 25 y 64 años es de 64,9\%, es decir casi dos de cada tres tienen sobrepeso/obesidad ${ }^{(14)}$. Existe una tendencia a mayor edad, mayor sobrepeso y obesidad (14).

La obesidad puede afectar en sí misma la función pulmonar, pero su relación con la EPOC ha sido poco investigada (21). El tabaquismo y la obesidad son los principales factores de riesgo de comorbilidades crónicas complejas, actúan sinérgicamente se asocian a resistencia a la insulina, estrés oxidativo, aumento de adipocitocinas y marcadores de la inflamación lo que conduce a inflamación endotelial y cardiovascular. Así la obesidad por sí misma agrega morbilidad a la enfermedad respiratoria y potencia otros factores de riesgo cardiovascular (22).

Diabetes miellitus (DM) es una defecto absoluto o relativo de la secreción y/o acción de la insulina a nivel periférico, según la Asociación Americana de Diabetes (ADA) la define como una glicemia plasmática en ayunas mayor o igual $126 \mathrm{mg} / \mathrm{dl}$ o una glicemia mayor o igual a $200 \mathrm{mg} /$ dl aleatoria en un paciente con síntomas clásicos de hiperglicemia o crisis de hiperglicemia ó en la prueba de tolerancia a la glicemia, luego de dos horas de la ingesta de la solución azucarada o una hemoglobina glicosilada mayor o igual a $6,5 \%{ }^{(23)}$. La prevalencia de diabetes en el Uruguay es del $8 \%{ }^{(14)}$. El estudio ECCO realizado en los servicios de medicina interna en España en pacientes con la EPOC que ingresaron por exacerbación aguda, mostró la presencia de DM en el $29,5 \%{ }^{(24)}$. La diabetes se asocia en forma independiente con la función pulmonar reducida y con la obesidad pueden empeorar aún más la gravedad de la EPOC ${ }^{(25)}$

La dislipemia se basa en los criterios séricos de Colesterol total menor de $200 \mathrm{mg} / \mathrm{dl}$, Trigliceridos menor de $150 \mathrm{mg} / \mathrm{dl}$, Colesterol-LDL menor de $130 \mathrm{mg} / \mathrm{dl}$ y Colesterol-HDL mayor de $50 \mathrm{mg} / \mathrm{dl}^{(14)}$. No está bien caracterizado el patrón de dislipemia en la EPOC, pero se ha visto mayor prevalencia de dislipemia en éstos pacientes que en los casos controles ${ }^{(26)}$

La EPOC es un factor de riesgo independiente para el desarrollo de cáncer broncopulmonar, varios estudios constituyen la base para tal afirmación, la incidencia de cáncer broncopulmonar es 2 a 5 veces superior en los pacientes fumadores con bronquitis crónica o enfisema comparado con una población de fumadores sin EPOC ${ }^{(27-29)}$. Luego de ajustar las variables de confusión, el riesgo es mayor en pacientes con enfisema, mayores de 60 años, índice paquete-año (IPA) mayor de 60 e IMC menor de $25^{(3)}$

El objetivo de este trabajo es conocer las características clínico-demográficas de una población de pacientes con EPOC asistidos en el servicio de Medicina Interna del Hospital Pasteur determinando las principales comorbilidades y sus características.

\section{Materiales y métodos}

Estudio observacional, descriptivo y trasversal en el período comprendido entre el $1^{\circ}$ de octubre de 2015 y el 31 de diciembre de 2016 que incluyó 61 pacientes captados durante la internación en el Servicio de Medicina Interna del Hospital Pasteur y controlados al alta en la policlínica de EPOC, durante el período señalado.

Criterios de inclusión: ambos sexos, mayores de 40 años, clínica y factores de riesgo para la enfermedad, tales como antecedentes de tabaquismo (activo, pasivo o ex tabaquista), exposición a biomasa, gases y polvos orgánicos e inorgánicos o diagnóstico previo de EPOC, con consentimiento informado firmado. 
Criterios de exclusión: deterioro cognitivo moderado-severo, pacientes postrados, embarazadas, tuberculosis pulmonar activa, enfermedades neuromusculares y pacientes que no consientan en participar de este estudio y/o no firmen el consentimiento informado.

Los pacientes que requirieron espirometría para el diagnóstico de la EPOC, se postergó la realización de la misma al menos 3 meses luego de la cirugía o procedimiento invasivo en el tórax.

Las siguientes variables cuantitativas fueron expresadas mediante la media y desvío estándar: edad, índice paquete - año, VEF1, número de internaciones por año.

Las siguientes variables cualitativas fueron expresadas mediante frecuencias absolutas y frecuencias relativas porcentuales: sexo, nivel de instrucción, historia de tabaquismo (si o no), bronquitis crónica (sí o no), otros factores de riesgo asociados (exposición a carbón, leña, polvos inorgánicos), comorbilidades (sí o no) ( HTA, alcoholismo, obesidad, dislipemia, DM, cardiopatía isquémica cáncer broncopulmonar, virus de inmunodeficiencia adquirida $(\mathrm{VIH})$, enfermedad renal crónica (ERC) y otras enfermedades respiratorias.

El procesamiento de los datos se hizo en el programa EPI-DATA versión 3.1 y PSPP

\section{Resultados}

En 42/61 pacientes que estuvieron en seguimiento, se pudo confirmar el diagnóstico de EPOC por espirometía previa o durante el período analizado.

La edad media fue de 63 años ( \pm 11 años), predominando en el sexo masculino (24 hombres vs. 18 mujeres, $57.1 \%$ vs. $42.9 \%$ respectivamente). La mayoría tenían un bajo nivel de instrucción, correspondiendo gran parte a los grupos de primaria incompleta (15 pacientes, 35.7\%) y primaria completa (15 pacientes, $35.7 \%$ ). El resto se dividieron entre secundaria incompleta (6 pacientes, $14.3 \%)$, secundaria completa (1 paciente, $2.4 \%$ ) y estudios de nivel terciario (1 paciente, $2.4 \%$ ).

\section{A) Factores de riesgo}

El principal factor de riesgo fue el tabaquismo (incluye a los tabaquistas activos y a los ex tabaquistas), presente en el $100 \%$ de los pacientes, seguido por la exposición a polvos inorgánicos (13 pacientes, 31\%), leña (8 pacientes, 19\%) y carbón (1 paciente, $2.4 \%$ ). Solamente 26 pacientes $(59.1 \%)$ presentaron al tabaquismo como único factor de riesgo, mientras que 11 pacientes $(26.2 \%)$ asociaron dos factores de riesgo y 5 pacientes $(11.9 \%)$ asociaron tres factores de riesgo. Ningún paciente asoció los cuatro factores de riesgo estudiados.

En cuanto a la historia de tabaquismo, de los 42 pacientes EPOC, 18 (42.9\%) eran ex tabaquistas, siendo 9 el promedio de años en abstinencia (mínimo 0 y máximo 40 años). El IPA promedio fue $81.9( \pm 59.8)$.

\section{B) Características clínicas de la población y espirométricas de la población}

El síntoma más frecuente fue la disnea (32 pacientes, 76.1\%); 23 (54.8\%) cumplían criterios diagnósticos de bronquitis crónica y 21 pacientes (50\%) asociaban bronquitis crónica y disnea.

El grado de disnea se clasificó siguiendo la escala de disnea del mMRC. La mayoría (21 pacientes, 50\%) tenía disnea grado 3, seguida de disnea grado 1 (8 pacientes, 19\%), disnea grado 0 (6 pacientes, 14.3\%), disnea grado 2 (2 pacientes, 4.8\%) y disnea grado 4 (1 paciente, $2.4 \%)$.

Más de la mitad de los pacientes $(26,61.9 \%)$ se comportaron como exacerbadores frecuentes, habiendo presentado dos o más exacerbaciones en el último año o una que hubiera requerido ingreso hospitalario. El resto (16 pacientes, 38\%) presentó una exacerbación - sin ingreso hospitalario o ninguna exacerbación en el último año.

Combinando los datos del grado de disnea con el número de exacerbaciones, se clasificaron los pacientes en los grupos A-D definidos por las Guías GOLD 2017. En la figura 1 se presenta la distribución de los pacientes analizados. 
Figura 1: Distribución de los pacientes analizados $(n=42)$ según las categorías GOLD 2017 (A-D)

\begin{tabular}{|c|c|c|}
\hline $\begin{array}{r}\text { Historia de } \\
\text { exacerbaciones }\end{array}$ & & \\
\hline $\begin{array}{r}-\quad \geq 20 \geq 1 \text { (con } \\
\text { ingreso hospitalario) }\end{array}$ & $\underset{9(21.4 \%)}{\mathbf{C}}$ & $\begin{array}{c}\text { D } \\
17(40.45 \%)\end{array}$ \\
\hline \multirow[t]{2}{*}{$\begin{array}{r}0 \circ 1 \text { (sin ingreso } \\
\text { hospitalario) }\end{array}$} & $\underset{5(11.9 \%)}{\mathbf{A}}$ & $\begin{array}{c}\text { B } \\
7(16.7 \%)\end{array}$ \\
\hline & $\begin{array}{r}\mathrm{mMRC}<1 \\
\mathrm{CAT}<10\end{array}$ & $\begin{array}{r}\mathrm{mMRC} \geq 2 \\
\mathrm{CAT} \geq 10\end{array}$ \\
\hline
\end{tabular}

La severidad de la limitación al flujo aéreo, se encontró que la mayoría de los pacientes EPOC estudiados pertenecían a los grupos GOLD 2 o limitación moderada (16 pacientes, $38.1 \%$ ) y GOLD 3 o limitación severa (15 pacientes, 35.7\%). Del resto, 6 pacientes (14.3\%) correspondían a la categoría GOLD 4 (limitación muy severa) y 2 pacientes (4.8\%) a la categoría GOLD 1 (limitación leve).

\section{C) Comorbilidades de la población}

De las comorbilidades indagadas, la que más se asoció a la EPOC fue la HTA, presentándose en 19 pacientes (45.2\%), más de la mitad (12 pacientes) tenían hipertensión severa y poca adherencia al tratamiento dietético y medicamentoso, 3 pacientes pertenecían a la categoría GLOD II, 12 pacientes a la GOLD III y 4 pacientes a la categoría GOLD IV.

Le sigue el alcoholismo (14 pacientes, 33.3\%), la obesidad (10 pacientes, 23.8\%) 2 pacientes, $2.4 \%$ ) pertenecían a la categoría GOLD I y 8 pacientes a la categoría GOLD II, la dislipemia (8 pacientes, $19 \%$ ) y la diabetes mellitus (6 pacientes, $14.3 \%)$.

Otras enfermedades respiratorias se asociaron en 6 pacientes (14.3\%) (Síndrome de apnea hipopnea del sueño (SAHOS) en 3 pacientes, asma, intersticiopatías, bronquiectasias un paciente en cada una de éstas patologías). En forma menos frecuente se determinó la asociación con cardiopatía isquémica (4 pacientes, 9.5\%), CBP (3 pacientes, $7.1 \%)$, infección por VIH (2 pacientes, $4.8 \%$ ) y ERC (1 paciente (Gráfica 1$)$

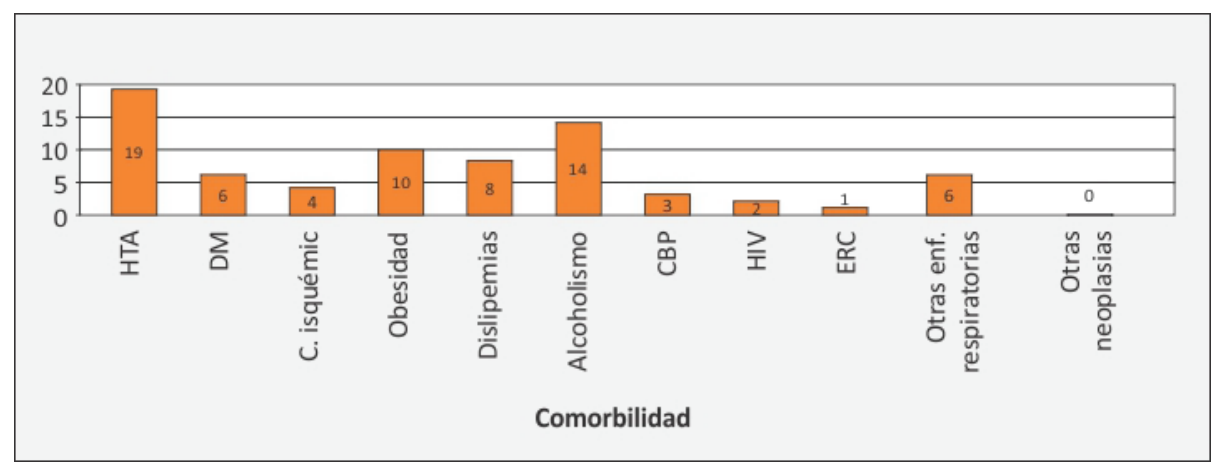

\section{Discusión}

La EPOC tiene una prevalencia de $19,7 \%{ }^{(29)}$ en Uruguay sin embargo, el número de pacientes captados es bajo, este hecho es de causa multifactorial.

En 9 de los 19 pacientes en los que la espirometría no cumplía los criterios diagnósticos para EPOC, presentaban clínica compatible y factores de riesgo para la enfermedad. Esta situación está bien descrita en el reciente estudio realizado por Meilan Han en la Universidad de Michigan, donde se analizaron 2700 pacientes incluyendo fumadores, ex fumadores y no fumadores, revisando síntomas respiratorios y espirometrías, encontraron que el $46 \%$ de los fumadores y ex fumadores tenían síntomas respiratorios similares a la EPOC, así como un mayor riesgo de exacerbación de los síntomas y consultas en el sistema de salud, aunque la espirometría era normal. Esta situación clínica no tiene nombre y no está claro si se trata de EPOC "temprana". Es necesario continuar la investigación sobre este grupo de pacientes que clínicamente tiene evidencia de enfermedad pero que no cumple los criterios espirométricos de la misma, para desarrollar protocolos terapéuticos ${ }^{(29)}$. 
De la población EPOC captada la edad media es de 63 años $( \pm 11)$, dato esperable dado el envejecimiento de la población en Uruguay y el aumento de la prevalencia de la enfermedad a medida que aumenta la edad. En el estudio EPI- SCAN realizado en distintas zonas de España, se describen las características sociodemográficas y clínicas de 3802 participantes de los cuales 386 padecían la EPOC, es decir una prevalencia de 10,2\%. La edad media era de 63,5 años $( \pm 10,5)$ similar a la de nuestro trabajo ${ }^{(30)}$. En el reciente estudio LEONPOC la edad de los pacientes fue de 72,8 años $\left( \pm 10,6\right.$ años) ${ }^{(31)}$.

En nuestro trabajo predominó el sexo masculino, como en los estudios internacionales. Esto se debe en parte a la mayor prevalencia del tabaquismo en el sexo masculino.

En IMC la media es de $25,79 \mathrm{~kg} / \mathrm{m} 2$ y la mediana (percentil 50) es 24,1 es interesante considerar la mediana, es decir un poco menos de mitad tiene sobrepeso-obesidad, lo que constituye una comorbilidad.

Predominó el bajo nivel educativo. Lo que tiene implicancias en la comprensión de la enfermedad, de las comorbilidades y en la adherencia al tratamiento. En las dos encuestas nacionales la población con menor nivel educativo (primaria o menos) fue la que tenía la mayor prevalencia de consumo de cigarros y tabaco y la que experimentó la menor disminución relativa de consumo de cigarrillos por día en la comparación con la población de otro nivel educativo (nivel secundario y terciario). Una posible interpretación es que las personas con menor nivel educativo integran en su mayoría sectores socio-culturales más vulnerables, a la vez que tienen mayores dificultades de acceso e interpretación de la información (32).

El principal factor de riesgo para el desarrollo de la enfermedad es el tabaquismo. En este trabajo todos los pacientes son tabaquistas o ex tabaquistas, hay un número considerable de pacientes que al alta de la internación abandonaron el hábito en base a las medidas educacionales, lo que constituye un éxito terapéutico. Pero aún por definición se consideraron en el grupo de los tabaquistas. El IPA promedio en nuestro trabajo fue $81.9( \pm 59.8)$ lo que traduce tabaquismo intenso. El IPA es más elevado que en los estudios nacionales e internacionales ${ }^{\left({ }^{(3)} \text {. Le sigue en }\right.}$ frecuencia la exposición a polvos inorgánicos vinculado a factores ocupacionales. El estudio Platino muestra la asociación entre la exposición a polvo en el lugar de trabajo y la EPOC. La mayor prevalencia $(22,9 \%)$ fue observada en los individuos que residían en Montevideo y trabajaron por 10 años o más en lugares con polvo ${ }^{(30)}$. En nuestro trabajo todos los pacientes procedían de zona urbana y la mayoría tenía el antecedente ocupacional. La evidencia reciente muestra que las exposiciones ocupacionales se asocian con peores resultados de la EPOC en fumadores, así se debe anticipar que la enfermedad es más grave en pacientes con exposición ocupacional ${ }^{(34)}$.

En la clínica el síntoma más frecuente es la disnea, seguida de la tos y la expectoración, similar al trabajo realizado por Miravitlles y Worth, un estudio observacional multinacional en 85 centros de diferentes países de Europa que reclutó 727 pacientes cuyo síntoma más frecuente fue la disnea seguido de la tos $(65,9 \%$ ) y la expectoración $(59,6 \%)$. En nuestro trabajo la disnea grado 3 de la clasificación mMRC era la más frecuente y en el trabajo de Miravitlles la más frecuente era la disnea grado 1 y 2 (33.6\% en cada caso) ${ }^{(35)}$.

Además $26(61.9 \%)$ pacientes se comportaron como exacerbadores frecuentes con dos o más exacerbaciones en el último año. En el trabajo antes citado, la mayoría $(53.9 \%)$ de los pacientes habían tenido solo una exacerbación en el año ${ }^{(35)}$. Es decir al comparar ambos trabajos el orden de frecuencia de los síntomas es similar, pero en el nuestro la severidad de la disnea y la frecuencia de exacerbaciones es mayor. Por tanto, al combinar los grados de la disnea con el número de las exacerbaciones la mayoría se encuentra en los grupos $\mathrm{D}$ y $\mathrm{C}$, de mayor severidad (Figura 1). Esta clasificación es posterior al trabajo de Miravitlles y por tanto no está referida por el autor.

La severidad de la limitación al flujo aéreo, según las guías GOLD 2017 (2) se determina a partir del valor del VEF1, clasificando la mayoría de los pacientes incluidos aquí como GOLD 2, lo que traduce una limitación al flujo aéreo moderada. Destacamos que si bien la mayoría de los pacientes son muy sintomáticos esto no se correlaciona con una obstrucción severa, como lo señala la literatura internacional ${ }^{(2)}$.

Hay varios estudios que muestran alta prevalencia de la patología cardiovascular en pacientes con la EPOC, así en el estudio CCECAN es un estudio trasversal en 300 pacientes con EPOC y en 524 sujetos del grupo control sin patología respiratoria, las dos poblaciones se compararon mediante análisis bivariado en cuanto al riesgo cardiovascular y mediante modelos de regresión logística se estimaron los riesgos en relación con la mortalidad cardiovascular de los pacientes con EPOC sobre el grupo control. Los pacientes con la EPOC presentaron alta prevalencia de HTA $(72 \%)$, obesidad (41\%), DM tipo $2(39 \%)$ y cardiopatía isquémica (16\%) desde estadios 
leves de la enfermedad. Respecto al grupo control, los pacientes con la EPOC presentaban un mayor riesgo de tener dislipidemia, diabetes tipo 2 y cardiopatía isquémica ${ }^{(36)}$.

La hipertensión arterial (HTA) es la comorbilidad más frecuente entre los pacientes con la EPOC de ambos sexos en nuestro trabajo y en los estudios internacionales ${ }^{(24-35-37)}$. Según datos recogidos por la Sociedad Europea de Hipertensión ${ }^{(10)}$, la coincidencia de ambas enfermedades podría afectar al 2,5\% de la población adulta. No están suficientemente explicados en la bibliografía los mecanismos patogénicos que unen la EPOC con la HTA, pero la hipoxia con el consiguiente incremento de radicales libres de oxígeno, daño endotelial y la estimulación de los ejes simpático y renina-angiotensina-aldosterona (RAA), así como factores inmunológicos e inflamatorios que rodean a la EPOC pueden contribuir a incrementar la prevalencia de la HTA en estos pacientes, hacer difícil el control y favorecer la aparición de enfermedad vascular aterosclerótica ${ }^{(38)}$.

Varios estudios intentaron vincular la HTA con la mortalidad, pero no se encontró evidencia que la hipertensión en pacientes con la EPOC tenga mayor mortalidad ${ }^{(9,24,39)}$.

Los resultados de diferentes estudios entre la HTA y el grado de severidad de la enfermedad son contradictorios. En el estudio PLATINO la hipertensión era más prevalente en las categorías más severas de la enfermedad (GOLD III y IV) ${ }^{(28)}$. El estudio realizado por López Varela y colaboradores encontraron la misma relación ${ }^{(28)}$. En nuestro trabajo también encontramos esta relación, así la mayoría de los pacientes con HTA pertenecían a las categorías GOLD III-IV, sin embargo hay estudios que muestran que en las categorías más severas de la enfermedad la frecuencia de la HTA disminuye drásticamente ${ }^{(15)}$.

La segunda comorbilidad más frecuente en este trabajo es el alcoholismo. No hay publicaciones que muestren resultados concluyentes entre EPOC y alcoholismo así como la frecuencia de esta asociación), la mayoría se centra en la relación entre el consumo de tabaco y alcohol ${ }^{(40)}$. Lo que si se estableció fue la influencia entre el consumo intenso de alcohol y la declinación de la función pulmonar (disminución del VEF1 y DLCO) cuya relación es directamente proporcional ${ }^{(40)}$.

En la intoxicación alcohólica aguda con depresión de conciencia y reducción del reflejo nauseoso, es posible que ocurra la aspiración de la vía aérea, lo que se asocia con riesgo de exacerbación de la EPOC ${ }^{(20)}$. En este trabajo ninguno de los pacientes presentó episodios de intoxicación alcohólica aguda y/o neumonía aspirativa que hubiera motivado el ingreso hospitalario

Existe una relación significativa entre EPOC y la presencia de alteraciones endocrinometabólicas como obesidad, dislipemia y diabetes. En nuestra población la DM se presentó en el $14,3 \%$ de los casos, algo menor a la hallada en dos trabajos realizados en España con población de pacientes EPOC hospitalizados en medicina interna, donde se comprobó una prevalencia de DM2 del 29,4\% en el estudio ECCO ${ }^{(24)}$ y de un $37 \%$ en el estudio ESMI (10). La obesidad tiene mayor prevalencia en pacientes EPOC leves a moderados (disminuye en EPOC severo) según estudios poblacionales realizados en Estados Unidos y España ${ }^{(41)}$.

En este trabajo la mayoría de los pacientes obesos pertenecían a las categorías GOLD I y II como en los estudios internacionales.

Los pacientes con EPOC y obesidad tienen más disnea, peor calidad de vida, utilizan más medicamentos inhalados y consumen más recursos sanitarios. La obesidad contribuye con la disminución de la capacidad torácica, aumento de la resistencia de la vía aérea y aumento del trabajo respiratorio ${ }^{(41)}$.

La dislipemia es también una comorbilidad frecuente, como se ve en estudio español ARCE que analiza esta asociación ${ }^{(42)}$

Por último en 6 pacientes del total de la muestra coexistía otra enfermedad respiratoria además de la EPOC, la más frecuente fue el SAHOS, la coexistencia de estas patologías empeora el pronóstico de ambos ${ }^{(2)}$. Estos pacientes eran muy sintomáticos y pertenecían a la categoría GOLD III.

En 3 pacientes coexistía la EPOC con el cáncer broncopulmonar, el diagnóstico se realizó en etapas avanzadas de ambas enfermedades, en pacientes muy síntomas.

Se conoce poco a cerca de la relación entre las comorbilidades y la mortalidad a corto plazo luego del alta hospitalaria por una exacerbación de la EPOC. El estudio ESMI se realizó en 70 servicios de Medicina Interna en España, es un trabajo Iongitudinal, observacional, 
multicéntrico en pacientes hospitalizados con exacerbación de la EPOC. Reunió 606 pacientes, las comorbilidades más frecuentes en los pacientes hospitalizados eran la HTA $(63,4 \%)$, la DM $(35,8 \%)$ y la dislipemia (34\%). El $4,5 \%$ de los pacientes (27 pacientes en total) murieron en los 3 meses siguientes luego del alta. Las conclusiones de este trabajo fueron que las comorbilidades son más frecuentes en los pacientes hospitalizados por la EPOC. Las comorbilidades se relacionan con la necesidad de hospitalización desde el servicio de urgencias, duración de la internación y reingresos. El número y tipo de comorbilidades se relaciona con el mal pronóstico vital a corto plazo ${ }^{(10)}$. En nuestro trabajo el $100 \%$ de los pacientes tenía por lo menos una comorbilidad y la mayoría una o más exacerbaciones al año lo que constituyen elementos del mal pronóstico, por tanto es necesario continuar con las medidas de prevención y control de los factores de riesgo para la EPOC así como el diagnóstico temprano y tratamiento de las comorbilidades y la EPOC

\section{Conclusiones}

La edad media y el sexo coinciden con los datos internacionales. Predominó el nivel de instrucción bajo y medio, lo que tiene implicancias en la comprensión de la enfermedad y las comorbilidades, asi como en la adherencia al tratamiento. El principal factor de riesgo para el desarrollo de la enfermedad es el tabaquismo. El 100\% de los pacientes tenían comorbilidades. La HTA fue la más frecuente coincidiendo con publicaciones internacionales y se asoció con las categorías más severas de la enfermedad. El alcoholismo es la segunda en frecuencia. Existe una relación significativa entre EPOC y la presencia de alteraciones endocrino-metabólicas como obesidad, dislipemia y diabetes. La obesidad sigue en frecuencia, éstos pacientes pertenecían a las categorías GOLD I y II como en los estudios internacionales. La presencia de otras enfermedades respiratorias constituyen un desafío diagnóstico. El número y tipo de comorbilidades se relaciona con el pronóstico vital a corto plazo cuando los pacientes son hospitalizados, en una exacerbación de la EPOC.

\section{Bibliografía}

1- Clin E.M, Beghé B, Fabbri L.M. Chronic obstructive pulmonary disease is just one component of the complex multimorbidities in patients with COPD. Am J Respir Crit Care Med. 2013; 187(7): 668-671.

2- Vogelmeiera CF, Crinerh GJ, Martínez FJ, Anzuetoc A, Barnesd PJ, Bourbeaue J, et al. Informe 2017 de la Iniciativa Global para el Diagnóstico, Tratamiento y Prevención de la Enfermedad Pulmonar Obstructiva Crónica: Resumen Ejecutivo de GOLD. Arch. bronconeumol. 2017; 53(3): 85-174.

3- Guía de Práctica Clínica para el Diagnóstico y Tratamiento de Pacientes con Enfermedad Pulmonar Obstructiva Crónica (EPOC) - Guía Española de la EPOC (GesEPOC). Arch. bronconeumol. 2017; 53 (supl 1): s1-s83.

4- Garin N, Koyanagi A, Chatterji S, Tyrovolas S. Olaya B, Leonardi M, et al. Global Multimorbidity Patterns: A Cross-Sectional, Population-Based Multi-Country Study. J Gerontol A Biol Sci Med Sci. 2016;71(2):205-14.

5- Rutten FH, Moons KG, Cramer MJ, Grobbee DE, Zuithoff NP, Lammers JW, et al. Recognising heart failure in elderly patients with stable chronic obstructive pulmonary disease in primary care: cross sectional diagnostic study. BMJ 2005; 331(7529):1379.

6- Le Jemtel TH, Padeletti M, Jelic S. Diagnostic and therapeutic challenges in patients with coexistent chronic obstructive pulmonary disease and chronic heart failure. J Am Coll Cardiol. 2007;49(2):171-80.

7- Donaldson GC, Hurst JR, Smith CJ, Hubbard RB, Wedzicha JA. Increased risk of myocardial infarction and stroke following exacerbation of COPD Chest. 2010;137(5):1091-7.

8- Estragó V, Alvarez P, coord. $3^{\circ}$ Consenso Nacional de Insuficiencia cardiaca [en línea]. Sociedad Uruguaya de Cardiología: Montevideo, 2013 [acceso enero 2019]. Disponible en: http://www.suc.org. uy/Consensos/3erConsensoNacionallnsuficienciaCardiaca_2013.pdf

9- Divo M, Cote C, de Torres JP, Casanova C, Marin JM, Pinto-Plata V, et al. Comorbidities and risk of mortality in patients with chronic obstructive pulmonary disease. Am J Respir Crit Care Med. 2012;186(2):155-61.

10- Almagro P, Cabrera FJ, Diez J, Boixeda R, Alonso Ortiz MB, et al. Comorbidities and short-term prognosis in patients hospitalized for acute exacerbation of COPD: the EPOC en Servicios de medicina interna (ESMI) study. Chest. 2012;142(5):1126-1133.

11- Pavasini R, d'Ascenzo F, Campo G, Biscaglia S, Ferri A, Contoli M, et al. Cardiac troponin elevation predicts all-cause mortality in patients with acute exacerbation of chronic obstructive pulmonary disease: Systematic review and metaanalysis. Int J Cardiol. 2015;191:187-93. 
12- Pavasini R, Biscaglia S, d'Ascenzo F, Del Franco A, Contoli M, Zaraket F, et al. Antiplatelet Treatment Reduces All-Cause Mortality in COPD Patients: A Systematic Review and Meta-Analysis. COPD. 2016 Aug;13(4):509-14.

13- Sociedad Uruguaya de Hipertensión Arterial. Hipertensión arterial: $3^{\circ}$ Consenso Uruguayo 凶1en línea]. Montevideo: SUC, 2005. Disponible en: http://www.suc.org.uy/pdf/consenso\%20ha3.pdf

14- Uruguay. Ministerio de Salud Pública. 2 a Encuesta Nacional de Factores de Riesgo de Enfermedades No Transmisibles. Montevideo: MSP, 2013.

15- Fumagalli G, Fabiani F, Forte S, Napolitano M, Marinelli P, Palange $\mathrm{p}$, et al. INDACO project: a pilot study on incidence of comorbidities in COPD patients referred to pneumology units. Multidiscip Respir Med. 2013;8(28):1-9.

16- Frei A, Muggensturm P, Putcha N, Siebeling L, Zoller M, Boyd CM, et al. Five comorbidities reflected the health status in patients with chronic obstructive pulmonary disease: the newly developed COMCOLD index. J Clin Epidemiol. 2014;67(8):904-11.

17- Sin DD, Man SF. Why are patients with chronic obstructive pulmonary disease at increased risk of cardiovascular diseases? The potential role of systemic inflammation in chronic obstructive pulmonary disease. Circulation. 2003; 107(11):1514-9.

18- Patel AR, Hurst JR. Extrapulmonary comorbidities in chronic obstructive pulmonary disease: state of the art. Expert Rev Respir Med. 2011; 5(5):647-62.

19- Rehm J, Baliunas D, Borges GL, Graham K, Irving H, Kehoe T, et al. The relation between different dimensions of alcohol consumption and burden of disease: an overview. Addiction. 2010; 105(5): 817-843.

20- Kuznetsov VN, Shelygin KV, Grjibovski AM, Mariandyshev AO, Johansson E, Bjune GA. Incidence of Tuberculosis and Associations with Indicators of Alcohol Consumption in Three regions of Northwest Russia in 1975-2009: A Time-Series Analysis. J Environ Public Health. 2013; 2013:693963.

21- Parameswaran K, Todd DC, Soth M. Altered respiratory physiology in obesity. Can Respir J 2006; 13 : 203-210.

22- Strong K, Mathers C, Epping-Jordan J, Beaglehole R. Preventing chronic disease: a priority for global health. Int J Epidemiol 2006; 35: 492-494.

23- American Diabetes Association. Standards of Medical Care in Diabetes 2011Standards of Medical Care in Diabetes-2011. Diabetes Care. 2011; 34(Suppl 1): S11-S61.

24- Almagro P, López García F, Cabrera FJ, Montero L, Morchón D, Díez J, et al; Grupo EPOC de la Sociedad Española de Medicina Interna. Estudio de las comorbilidades en pacientes hospitalizados por descompensación de la enfermedad pulmonar obstructiva crónica atendidos en los servicios de medicina interna: Estudio ECCO. Rev Clin Esp. 2010;210:101-8

25- Litonjua AA, Lazarus R, Sparrow D, Demolles D, Weiss ST. Lung function in type 2 diabetes: the Normative Aging Study. Respir Med 2005; 99: 1583-1590.

26- Naik D, Joshi A, Vizhali TP, Thomas N. Chronic Obstructive Pulmonary Disease and the Metabolic Syndrome: Consequences of a Dual Threat. Indian J Endocrinol Metab. 2014; 18(5): 608-616.

27- Sakamoto Y, Yamauchi Y, Yasunaga H, Takeshima H, Hasegawa W, Jo T, Sasabuchi Y, et al. Development of a nomogram for predicting in-hospital mortality of patients with exacerbation of chronic obstructive pulmonary disease. Int J Chronic Obstr Pulm Dis. 2017; 12: 1605-1611.

28- López Varela MV, Montes de Oca M, Halbertc R, Muiño A, Tálamo C, Pérez-Padilla R, et al. Comorbilidades y estado de salud en individuos con y sin EPOC en 5 ciudades de América Latina: Estudio PLATINO in Five Latin American Cities : The PLATINO Study. 2015;49(11):468-74.

29- Woodruff PG, Barr, RG, Bleecker E, Christenson SA, Couper D, Curtis JL, et al. Clinical significance of symptoms in smokers with preserved pulmonary function. N Engl J Med 2016; 374:1811-1821.

30- J. Ancochea C, Badiola E. Duran-Tauleria F. Garcia Rio M, Miravitlles L. Muñoz V. et al. Estudio EPISCAN: resumen del protocolo de un estudio para estimar la prevalencia de EPOC en personas de 40 a 80 años en España. Arch Bronconeumol. 2009;45(1):41-47.

31- García García S, Carazo Fernández L, García JJ, Naveiro Rilo JC. Enfermedad pulmonar obstructiva crónica: los pacientes en la vida real. Estudio LEONPOC. Aten Prim. 2017. http://dx.doi.org/10.1016/j. aprim.2017.01.00 
32- Abascal W, Esteves E, Goja B, González F, Lorenzo A, Sica A. Impacto de las políticas de control de tabaco en el Uruguay. 2006-2009.: Programa Nacional para Control del Tabaco. Arch. Med Int. 2013; 35 ( Suppl 4 ): 1-16.

33- Uruguay. Junta Nacional de Drogas. Secretaría Nacional de Drogas; Centers for Disease Control and Prevention; Organización Panamericana de la Salud; Organización Mundial de la Salud. Encuesta Mundial de Tabaquismo en Jóvenes (GYTS). Informe de investigación. Uruguay [en línea]. 2006 [acceso enero 2019]. Disponible en: http://www.who.int/whosis/whostat2006

34- Hong Y, Lim MN, Kim WJ, Rhee CK, Yoo KH, Lee JH, et al. Influence of Environmental Exposures on Patients with Chronic Obstructive Pulmonary Disease in Korea. Tuberc Respir Dis (Seoul). 2014;76(5):226-32.

35- Miravitlles M, Worth H, Soler Cataluña JJ, Price D, De Benedetto F, Roche N et al. Observational study to characterise 24-hour COPD symptoms and their relationship with patient-reported outcomes: results from the ASSESS study. Respir Res. 2014; 15:122.

36- Figueira Gonçalves JM, Dorta Sánchez R, Rodríguez Pérez MC, Viña Manrique P, Díaz Pérez D et al. Comorbilidad cardiovascular en pacientes con enfermedad pulmonar obstructiva crónica en Canarias (estudio CCECAN) Clin Investig Arterioscler. 2017;29(4):149---156

37- Gómez Antúnez M, Cabrera Aguilar F.J, Muiño Míguez A. La EPOC como enfermedad sistémica: comorbilidades más frecuentes.Madrid: Sociedad Española de Medicina Interna, 2014. pp. 1-5.

38- Miller J, Edwards LD, Agustí A, Bakke P, Calverley PM, Celli B, et al. Comorbidity, systemic inflammation and outcomes in the ECLIPSE cohort. Respir Med. 2013;107: 1376-84.

39- Sin DD, Anthonisen NR, Soriano JB, Agusti AG. Mortality in COPD: The role of comorbidities. Eur Respir J. 2006 Dec;28(6):1245-57.

40- Frantz S, Wollmer P, Dencker M, Engström G, Nihlén U. Associations between lung function and alcohol consumption--assessed by both a questionnaire and a blood marker. Respir Med. 2014;108(1):114-21.

41- Monteiro F, Camillo CA, Vitorasso R, Sant'Anna T, Hernandes NA, Probst VS, et al. Obesity and physical activity in the daily life of patients with COPD. Lung. 2012;190:403-10.

42- Tamimi A, Serdarevic D, Hanania NA. The effects of cigarette smoke on airway inflammation in asthma and COPD: therapeutic implications. Respir Med. 2012;106:319-28.

\section{Aporte cada autor al trabajo}

Selene Correa: 15\%. Redacción.

Mariana Gonzaléz: 15\%. Redacción.

Sofía de Betolaza: 15\% Redacción.

Cecilia Spiess: $15 \%$. Metodología.

Patricia Perera: $15 \%$. Recolección datos.

Santiago Algorta: 15\%. Recolección datos.

Mabel Goñi: 10\%. Corrección general. 